\title{
Souvenirs des morts : techniques de gestion de la mémoire dans un village de Nouvelle-Guinée
}

\section{Roger Ivar Lohmann}

\section{(2) OpenEdition \\ 12 Journals}

Édition électronique

URL : http://journals.openedition.org/jso/748

DOI : $10.4000 /$ jso. 748

ISSN : $1760-7256$

Éditeur

Société des océanistes

\section{Édition imprimée}

Date de publication : 1 juin 2007

Pagination : 45-57

ISBN : 978-2-85430-010-9

ISSN : 0300-953x

\section{Référence électronique}

Roger Ivar Lohmann, «Souvenirs des morts : techniques de gestion de la mémoire dans un village de Nouvelle-Guinée ", Journal de la Société des Océanistes [En ligne], 124 | Année 2007-1, mis en ligne le 01 juin 2010, consulté le 01 mai 2019. URL : http://journals.openedition.org/jso/748 ; DOI : 10.4000/ jso.748 


\title{
Souvenirs des morts : techniques de gestion de la mémoire dans un village de Nouvelle-Guinée ${ }^{1}$
}

\author{
par
}

Roger IVAR LOHMANN*

\section{RÉSUMÉ}

Les Asabano de Papouasie Nouvelle-Guinée traitent de diverses façons les objets considérés comme des "mémentos » de leurs compagnons décédés. Plusieurs cas sont abordés ici, notamment la conservation à long terme de ces objets, leur destruction immédiate et leur abandon à la désintégration. Le choix de la stratégie qui va être suivie par les survivants est influencé par divers facteurs, parmi lesquels la valeur pragmatique et sentimentale accordée à l'objet et l'état émotionnel du moment. Toutefois, le facteur décisif va être le type de souvenir que le survivant cherche à conserver de la relation qu'il entretenait avec le défunt. Le soin apporté à l'objet correspond au désir de conserver un souvenir cher, alors que sa destruction vise à expurger une mémoire douloureuse. La vitesse à laquelle un souvenir est détruit reflète le degré auquel on aspire à oublier et à aller de l'avant. Ces attitudes reflètent explicitement le fait que des objets peuvent évoquer les souvenirs de relations passées et il est utile d'aborder chaque stratégie comme une forme de gestion de la mémoire.

MoTS-CLÉs : mémoire, mémentos, souvenirs, rituel mortuaire, deuil, culture matérielle.

Lorsque je réalisais mon travail de recherche sur le terrain auprès des Asabano en 1994-1995, j'ai été surpris, en tant que Nord-Américain descendant de Nord-Européens, par la façon dont ils traitaient les objets associés à des compa-

\begin{abstract}
The Asabano of Papua New Guinea treat objects regarded as mementos of deceased companions in a variety of ways. Several cases are considered here, including long-term retention, immediate destruction, and decay through exposure. Which strategy survivors follow is influenced by various factors, including the pragmatic and sentimental value of the item and one's emotional state at the time. The primary motivating factor, however, is the type of memory that the survivor wishes to retain of the former living relationship. Curation corresponds with the desire to retain a cherished memory, while destruction correlates with expunging a painful memory. The speed with which a memento is destroyed reflects the rate at which one hopes to forget and move on. These responses reflect the recognition that objects can evoke memories of past relationships, and it is useful to regard each strategy as a form of memory management.
\end{abstract}

KEYWORDS: memory, mementos, souvenirs, mortuary ritual, mourning, material culture

gnons décédés. Un arbre fruitier productif fut coupé après la mort d'une jeune mère. Les vêtements d'un fils mort furent accrochés à un arbre. L'étui pénien d'un père décédé fut conservé durant des décennies puis vendu. Ces actions

1. Des versions antérieures de cet article furent présentées aux rencontres de la Society for the Anthropology of Religion à Vancouver, Colombie-Britannique (Canada) en avril 2005, et de la European Society for Oceanists à Marseille (France), en juillet 2005. Mes remerciements à Antoine Lassagne, pour avoir traduit le manuscrit de l'anglais, à Heather M.-L. Miller et Eric Venbrux pour leur aide et leurs réactions à ces idées, et à Pierre Lemonnier pour tous ses apports à l'édition finale du manuscrit.

* Department of Anthropology, University of Trent, Canada, rogerlohmann@trentu.ca 
étaient de toute évidence l'expression de quelque chose, mais de quoi ? Cet article propose des réponses aux questions qui occupaient mon esprit quand j'ai commencé à observer ces comportements. Je décris chaque cas entourant la production de souvenirs lors des décès survenus quand j'étais sur le terrain, y compris les discours, les rituels et autres comportements. Ce faisant, les coutumes funéraires asabano et les attitudes adoptées envers les mémentos (qui constituent un type précis de culture matérielle) sont dégagées au travers de l'examen de cas particuliers qui étaient à la fois appropriés culturellement et individuellement distinctifs. En bref, mon analyse vise des stratégies de gestion de la mémoire qui sont un dénominateur commun à tous ces cas, ainsi que des effets de mémoire spécifiques et recherchés, en tant qu'ils motivent et sous-tendent chaque cas particulier.

La vie sociale avec des compagnons construit et met en scène des souvenirs. Lors de la perte d'un compagnon, on se retrouve seul avec les vifs souvenirs de moments qui furent auparavant autant construits que partagés de façon conviviale. Les événements qui causent la perte, en particulier la mort, génèrent un souvenir nouveau et désagréable qui se retrouve associé schématiquement aux souvenirs agréables qui furent construits et entretenus par association et nostalgie tout au cours de la relation avec le défunt. Le deuil et le retour à l'équilibre émotionnel qui suit après coup - comme le degré et la vitesse auxquels ils vont se faire - sont en grande partie une question de gestion de la mémoire, dans laquelle on doit assumer seul, isolé physiquement du défunt, ce qui auparavant était une tâche mutuelle, pour arriver à accepter le souvenir de la perte et des rappels de l'absence. Ironiquement, une façon possible de favoriser la gestion de la mémoire dans cette situation est de focaliser la conscience et l'action sur les rappels euxmêmes - sur les mémentos ou marqueurs matériels qui évoquent les souvenirs de la personne perdue, les événements partagés et l'expérience de la perte. Dans les exemples de ce phénomène que j'ai observé parmi les Asabano, les objets laissés par les compagnons décédés servaient de point de mire aux survivants pour gérer leurs souvenirs des défunts. Traiter les mémentos différemment permettait aux survivants d'exprimer ces souvenirs et, en même temps, de décider de la façon dont ces souvenirs seraient rappelés dans le futur. Les mémentos servaient de symboles des relations passées avec certains compagnons en particulier et, de la même manière que les compagnons gèrent leurs souvenirs communs au travers des conversations et de la nostalgie, la mani- pulation des mémentos des compagnons décédés permettait aux survivants de poursuivre ce travail perpétuel de mémoire et de supporter le trauma causé par la perte de ces compagnons.

Comme l'a observé Mihaly Csikszentmihalyi (1993: 23) :

«objects reveal the continuity of the self through time, by providing foci of involvement in the present, mementos and souvenirs of the past, and signposts to future goals.»

Les objets impliqués dans la gestion des souvenirs des morts existent dans une stupéfiante gamme d'échelles, qu'on en considère la taille (depuis des monuments gigantesques jusqu'aux minuscules bibelots), l'ampleur sociale (obsèques de groupes ou d'individus), la signification historico-mythologique (des trésors nationaux jusqu'aux restes plébéiens), la relation qu'ils soulignent avec le défunt (des proches de celui-ci jusqu'à des gens qui ne l'ont jamais rencontré) et la temporalité mise en avant (du passé lointain jusqu'à un passé proche) (Hallam et Hockey, 2001 : 7). Quelle qu'en soit l'échelle, les mémentos ne sont pas seulement des marqueurs du passé. Ils aident à la construction et à l'accomplissement de soi dans le présent continuel qui est, en définitive, l'instant hanté par les souvenirs.

\section{Les Asabano et leurs mémentos}

Au nombre d'environ deux cents, les Asabano pratiquent en majorité une horticulture de subsistance et vivent en hameaux dans les vastes forêts montagneuses situées à l'est de la piste d'atterrissage de la mission de Duranmin, dans la province de Sandaun, en Papouasie NouvelleGuinée. Leur langue papoue, unique, est classée dans le phylum Sepik-Ramu. Leur premier contact eut lieu avec une patrouille coloniale australienne en 1963, puis leur missionnarisation par des Telefolmin voisins provoqua leur conversion massive au christianisme baptiste en 1977. Lors de mes séjours de recherche auprès d'eux en 1991, 1994-1995 et 2005, j'ai documenté nombre de leurs coutumes pré-contact, le contact lui-même et l'histoire de leur conversion, les raisons pour lesquelles ils adoptèrent des idées religieuses étrangères, et leur situation actuelle (voir Lohmann, 2000b, 2001, 2003b, 2007).

Les Asabano sont liés tous ensemble par des rhizomes de consanguinité et d'affinité comme une seule et même famille étendue. Outre la parenté et le mariage, leurs relations sont enrichies et entretenues par des souvenirs d'expé- 
riences communes imprimés dans l'esprit. En Asaba, on les désigne par sosa mablele ou « pensées de l'âme, sentiments ». En Tok Pisin, ce genre de préoccupation à propos d'une personne est appelé wari (dérivé de l'anglais worry). Ces émotions s'intensifient en l'absence d'un compagnon. Les gens remplacent souvent la présence physique d'un compagnon manquant par des objets ou des lieux qui évoquent de bienveillants sosa mablele. Des objets fabriqués ou utilisés par la personne sont particulièrement adaptés à ce propos. Ils sont appelés en Asaba bedele et en Tok Pisin ol memori (dérivé de l'anglais memories) ou "mémentos". Des biens ou des effets personnels peuvent être sollicités pour servir de mémentos auprès d'un compagnon qui va s'absenter pour un temps. En Asaba, un « mémento appartenant à Seli » sera désigné par bebe Seli yobu et, en Tok Pisin, il serait nommé memori bilong Seli.

Doug Dalton a observé un usage similaire de mémentos, également appelés ol memori chez les Rawa, un autre peuple de Papouasie NouvelleGuinée. Selon lui, les mémentos « serve only to remind the recipient of the person who left it behind and the fact that this person gave it » et les Rawa définissent la mémoire «as an experience of loss rather than a technique of preservation » (Dalton, $2001:$ 125, 126). Ceci soulève deux points importants. Premièrement, tant que les mémentos sont reçus en échange, ils évoquent toute l'importance sociale pour laquelle les dons sont reconnus, notamment en Mélanésie. Et quand ces objets sont conservés plutôt qu'échangés de nouveau, ils connectent celui qui en a la charge aux relations passées auxquelles leurs identités profondes renvoient (Godelier, 1999[1996] : 200). Deuxièmement, Doug Dalton nous rappelle que les mémentos servent non seulement à se souvenir, mais aussi à contrôler l'oubli.

Un départ et une séparation engendrent du chagrin et un hiatus dans une relation de compagnie. Quand meurent des individus, qu'arrive-t-il à ce réseau de relations et d'expériences communes qui relient entre eux les Asabano, soudés par le souvenir ? Dans la perspective coutumière, la mort entame mais ne rompt pas automatiquement ces connexions. Alors que des compagnons sont biologiquement (mais pas forcément spirituellement) emportés par la mort, les cadavres et les mémentos restent pour évoquer leur mémoire, pour que les survivants s'en rappellent. Ces témoignages fournissent non seulement des jalons potentiels aux relations imaginées en cours, mais aussi aux expériences communes dont on se souvient et qui se poursuivent.
Il y a cent ans, Robert Hertz (1960 [1907]) suggérait que, dans certains rituels mortuaires, l'état du corps symbolise la position sociale et existentielle de l'âme de la personne décédée. Il observa que c'est particulièrement le cas dans les doubles funérailles, au cours desquelles on pratique une seconde inhumation. La chair temporaire, humide et impure est laissée à pourrir jusqu'à disparaître, alors que les os, permanents, secs et propres sont ramassés et conservés pour refléter le nouveau statut d'ancêtre qu'acquiert l'âme décédée ; pour garder un souvenir positif des défunts et pour leur conférer un rôle parmi les vivants. J'ai précédemment prolongé l'idée de Robert Hertz en montrant que, dans les pratiques mortuaires asabano, des traitements spécifiques du corps (incluant le soin, le rejet et la destruction des parties non périssables) reflètent non seulement les croyances à propos de la condition de l'âme de la personne décédée, mais aussi le type de relations futures que les survivants veulent conserver avec le défunt. Ces croyances conduisaient les survivants à manipuler les corps de différentes façons (Lohmann, 2005).

Dans le présent article, j'ai déplacé mon attention, précédemment portée sur les corps morts, vers ces objets laissés par les défunts et que les survivants considèrent comme des mémentos: objets qui se confondent avec les choses considérées comme faisant partie de l'héritage de quelqu'un, mais qui sont principalement évalués sur une base sentimentale plutôt qu'économique. Peut-on transposer l'argumentation de Robert Hertz portant sur les corps au cas des mémentos ? Chez les Asabano tout au moins, la fonction culturelle et psychologique des mémentos est différente de celle des corps. Bien plus que le simple constat, le signifiant ou la cause de l'état actuel ou désiré de l'âme d'une personne décédée, ou de la relation entre les vivants et les morts, le fait de traiter les mémentos différemment correspond à diverses formes de gestion de la mémoire. Les mémentos ne représentent pas seulement la personne décédée, ils rappellent des événements relationnels impliquant cette personne. Dans la pensée des Asabano, conserver les os d'une personne décédée maintient une relation d'échange spirituel avec elle. Au contraire, la conservation d'un mémento ne peut maintenir la relation elle-même, mais elle a le pouvoir de rappeler un des souvenirs heureux d'une relation passée. Alors que détruire un cadavre en le jetant dans une rivière est entendu comme un acte visant à annihiler l'âme de la personne décédée, coupant ainsi court à quelque relation future possible, détruire un mémento 
aide à effacer le souvenir doux-amer du défunt. Détruire des mémentos ne nuit pas à l'âme de la personne décédée; de même, en prendre soin ne permet pas de maintenir une relation de la même façon que prendre soin d'ossuaires le permet traditionnellement. Les événements sociaux que les mémentos évoquent appartiennent au passé, plutôt qu'au présent ou au futur. De plus, contrairement aux cadavres, ces objets n'impliquent pas de rites de passage relatifs au statut social de la personne décédée, comme en en trouve dans les funérailles dayak analysées par Robert Hertz et les pratiques mortuaires asabano. Dans la mesure où ces mémentos sont manipulés ou mis au centre d'un rituel, c'est la mémoire de la relation passée $\mathrm{du}$ survivant-propriétaire qui est chérie ou expurgée, plutôt que la relation actuelle avec le défunt.

J'en viens maintenant aux trois exemples mentionnés dans l'introduction. Je me suis d'abord rendu compte une pratique qui consiste à conserver des mémentos lorsque les gens me proposaient à l'occasion de me vendre de vieilles affaires de proches morts il y a longtemps. Dans mon cas, il s'agissait d'un tambour, de bijouterie faite de défense de cochon et de parures en coquillage, y compris plusieurs vieux étuis péniens. Le premier exemple concerne un de ces cas. Étant donné que la personne à qui le mémento appartenait à l'origine était morte depuis longtemps, je ne peux donner d'information sur ses funérailles. De plus, le souvenir de l'actuel propriétaire du mémento s'était en partie érodé avec le temps. Les deux autres exemples impliquent des personnes décédées que j'avais connues personnellement et je suis donc en mesure de commenter leurs funérailles et de décrire la façon dont les gens traitaient les mémentos alors que la perte $\mathrm{du}$ proche était encore fraîche et intense. Mes comptes rendus tout comme l'expression des émotions des individus sont donc nécessairement dépendants du temps écoulé depuis la mort.

\section{Le soin : l'étui pénien d'un vieil homme}

L'habillement traditionnel des Asabano se compose, pour les femmes, d'une jupe composée de couches d'herbes et, pour les hommes, d'un étui pénien généralement confectionné à partir d'une calebasse ou d'une portion de coquillage acquis par le biais des réseaux d'échanges avec les populations de la côte. Contrairement aux calebasses, les coquillages servant d'étui phallique sont durables et plus beaux et, par consé- quent, plus précieux. À la fin du mois d'avril 1994, c'est-à-dire vers le début de mon principal séjour de terrain, un vieil homme nommé Madfe apporta l'étui pénien de son père Mesisi et offrit de me le vendre pour six kina (environ 6,50 dollars américains à l'époque). Son père était mort quelque trente ans auparavant, peu après le premier contact avec les Européens en 1963, dans l'ancien hameau de la rivière Kienu, qu'ils avaient pour cette raison abandonné en 1972. Madfe me conta qu'il avait gardé le coquillage comme souvenir de son père. On m'en avait déjà offert un à acheter auparavant et, à cette époque, je commençais à penser que je gaspillais mes maigres ressources dans des sous-vêtements moisis. Je me sentais aussi mal à l'aise à l'idée de départir les gens de leurs biens familiaux ; mais ceux qui me les offraient, eux, étaient davantage anxieux vis-à-vis des morts. Je me consolais en pensant que si j'en prenais la charge, ils seraient préservés et finalement déposés dans un musée.

Avant le premier contact, les Asabano étaient semi-nomades, changeant de hameaux à peu près tous les vingt ans, au moment où les sites devenaient insalubres, lorsque les forêts environnantes étaient vidées de leur gibier et/ou quand les jardins nécessitaient d'être mis en jachère. Aujourd'hui, tout comme autrefois, les gens ne possèdent pas beaucoup de biens matériels et il y a peu d'objets dont ils prennent soin et qu'ils conservent plus de quelques années. $\mathrm{Ce}$ mémento représentait quelque chose que Madfe voulait conserver, mais dont il aurait tout autant pu se départir si une opportunité d'échange valant la peine se présentait. Madfe se sentit prêt à céder le mémento de son père après trente années pour le prix de quelques kilos de riz et d'une boîte de conserve de poisson; le genre de chose qu'il aurait pu se procurer avec ses six kinas. Il ne semblait pas triste, ni même pensif, à l'idée de s'être séparé du coquillage ; peut-être était-ce un réconfort de savoir qu'il serait conservé quelque part, au côté des histoires que j'avais collectées sur son peuple. Après tout, il savait que mon intention était de continuer de l'utiliser comme mémento de lui, de son père, de son peuple. Il serait difficile de donner une valeur monétaire à un objet d'un tel usage. En même temps, je m'interrogeais sur le sens que Madfe avait de la valeur de l'objet, sentimentale comme matérielle, mais je n'étais pas encore préparé à poser directement de telles questions. Néanmoins, des indices généraux allaient apparaître sous peu.

Cinq mois après que j'avais acheté le vieil étui pénien, des hommes vinrent me questionner au 
sujet d'une machette qu'un aîné téléfolminasabano nommé Amtoseng avait acquise auprès de moi. Lui avais-je donnée ou bien vendue? Je leur répondis que je la lui avais vendue pour trois kina (ce qui correspondait à peu près à la moitié du coût d'une machette neuve). Pour me justifier et pour que tout le monde ne s'attende pas à ce que je procure des machettes au même prix, j'expliquais qu'elle était usagée - je l'avais payée six kina neuve et ne l'avais utilisée que quelques mois. Les hommes trouvèrent cela très drôle, et m'expliquèrent qu'ils considèrent que les vieilles choses ont beaucoup plus de valeur à cause de l'attachement sentimental qu'on leur porte. La machette avait pris et non perdu de la valeur à cause des souvenirs qu'elle pouvait évoquer $\mathrm{du}$ temps où je l'utilisais. Pour son nouveau propriétaire, parce que j'avais utilisé cette machette, celle-ci devait avoir une plus grande valeur car elle pourrait tenir lieu de mémento de moi. Établir la valeur d'un mémento est une chose complexe à l'intérieur d'une même culture, tout comme ça l'est à l'intérieur de l'esprit d'une seule et même personne. Pour faire un travail approfondi, nous devons, comme David Graeber (2001) l'expose «see how meaning [...] turns into desire». De combien se complique l'équation quand un échange interculturel de valeur sentimentale est en jeu?

Pour revenir à l'étui pénien de Madfe, il s'agissait d'un objet portable, toujours fonctionnel, et qui pouvait évoquer la mémoire de son père décédé il y a si longtemps. Il l'avait conservé toutes ces années pour l'aider à se souvenir de son père, mais sa disparition remontait à si loin, et Madfe étant lui-même aujourd'hui un vieil homme, que l'objet n'apparaissait plus aussi nécessaire comme mémento. En tout cas pas au point qu'il ne pût être cédé pour une petite contrepartie, tout en sachant que cela me ferait plaisir et lui donnerait une nouvelle vie en tant que mémento. Ainsi, prendre soin du mémento permit de garder vivante une mémoire chérie pour des décennies, bien qu'il eût pu servir à d'autres usages. Dans le cas suivant, les mémentos d'une mort plus récente et plus tragique sont placés à l'extérieur, où ils peuvent être soit vus, soit ignorés et abandonnés à une lente désintégration. On peut discerner dans cette action une forme différente de gestion de la mémoire.

\section{Abandonner à la décomposition : les vêtements d'un petit enfant}

En septembre 1994, un petit enfant nommé Waniol contractait une maladie neurologique - méningite ou malaria cérébrale - et mourut en quelques jours. Un soir, Yalowad, le chef du village, vint chez moi et m'annonça que Waniol était mort en milieu de matinée. À environ cinq heures de l'après-midi, $\mathrm{j}$ 'entendais deux femmes pleurer, avec leurs vocalises caractéristiques. À six heures trente, au crépuscule, j'entendais clouer son cercueil. Le matin suivant, je vins à la grande maison communautaire, un bâtiment construit en matériaux de brousse qui est au centre de la vie du village. Beaucoup de villageois $\mathrm{y}$ avaient passé la nuit à veiller le corps.

La pratique de conserver et de veiller le cadavre dans la maison communautaire entre une et trois nuits de suite fut introduite par les missionnaires téléfolmin qui avaient converti les Asabano vingt ans auparavant. La coutume asabano traditionnelle, qui fut interdite par la loi coloniale australienne, consistait à exposer aussi vite que possible le corps sur une plateforme loin à l'écart du village et des sentiers. Les Asabano craignent que les sorcières ou les esprits belliqueux, qu'ils considèrent comme responsables de toutes les morts, soient attirés par le cadavre, dans l'espoir de l'opportunité de dévorer le " gibier » qu'ils ont chassé et tué ; et si le corps est gardé trop proche du village, ils pourraient tuer les petits enfants. Les gens éloignent donc le cadavre le plus rapidement possible afin de minimiser les risques. Certains expliquent que la récente coutume d'exposer le cadavre en évidence dans la maison communautaire est désormais possible parce que c'est maintenant la «bonne époque » et que Dieu tout puissant est là et les protège. Adopter cette nouvelle coutume semble être une démonstration de foi.

Alors que nous attendions le début du service funéraire, trois hommes âgés m'exposaient leurs théories, avançant que le garçon avait probablement été tué par des sorcières venant d'un groupe voisin à propos d'un vieux conflit. $\mathrm{La}$ mère du petit garçon était morte des années auparavant et son père, veuf, Pastor Wani, avait donné beaucoup d'amour à son fils. Ils étaient souvent venus ensemble me rendre visite dans ma maison. Quand son fils est mort, Wani était absent, en visite dans la ville minière de Tabubil. Wani prit l'avion pour rentrer et arriva au milieu de l'après-midi. Il entra dans la maison et serra la main de tous les adultes, le visage vide de toute expression. Il s'approcha ensuite du cercueil, qui avait été placé entre deux foyers. Il resta à sangloter, sans chanter, ni prononcer un mot pendant environ cinq minutes. Yowe, la femme qui s'était occupée de Waniol en l'absence de son père pleurait mélodieusement, en retrait. Toutes les autres personnes présentes étaient plus ou 
moins silencieuses. Deux jeunes femmes servaient de la nourriture - elles n'en proposèrent pas à Pastor Wani. Après un moment, Wani prit un nouveau drap de lit qu'il avait acheté à Tabubil, souleva Waniol de son cercueil et, alors que deux hommes l'aidaient à envelopper le corps dans le drap et à le replacer dans le petit cercueil, il se mit à sangloter de nouveau. Suivirent deux prières de groupe, pendant lesquelles Wani ferma les yeux comme c'est de coutume dans les prières chrétiennes locales. Mais contrairement aux autres, il ne prononça ni ne chuchota un mot.

Je me sentais malheureux aux funérailles et m'estimais partiellement responsable de la mort de l'enfant. Dans ses derniers jours, j'avais insisté pour qu'il soit envoyé à l'hôpital, mais mon conseil n'avait pas été pris en compte parce qu'un medium de l'Esprit saint avait eu une vision qui indiquait que le petit garçon était condamné. Étant non-croyant, je me demandais si je n'aurais pas dû intervenir plus fermement en dépit de nos différences culturelles.

Après les funérailles, un aîné nommé Sumole mentionna que les femmes pleurent généralement davantage que les hommes à la mort de quelqu'un. " Les femmes peuvent pleurer » ditil, «mais les hommes ne doivent pas pleurer quand quelqu'un est mort, parce qu'alors les sorcières le frapperont lui aussi ». A ces funérailles, je vis trois hommes pleurer, mais Yowe, qui avait gardé Waniol, resta à gémir mélodieusement plus longtemps que tout le monde. La mort de cet enfant chéri, qui faisait l'enchantement de tous, semblait ébranler fortement les gens, bien qu'ils craignissent clairement de montrer leurs émotions, en particulier celles du désespoir qui invite à une attaque spirituelle.

Je pensais à la mort d'un petit enfant, qui, dans toute culture, entraîne des événements caractéristiques. Les Asabano que j'ai connus considéraient les enfants comme particulièrement vulnérables à la mort et aux maladies causées par des esprits. Pour cette raison, ils gardaient du mieux qu'ils pouvaient les enfants éloignés des dépouilles des morts sur lesquels leurs esprits auraient pu faire une fixation. Les enfants et les nourrissons qui perdent leur mère sont dans une position particulièrement vulnérable, car l'esprit de la mère est supposé rechercher la compagnie de l'enfant. Ils ne peuvent être réunis que par la mort de l'enfant. L'enfant doit être complètement séparé de sa mère décédée car les sorcières et les esprits dévorent le corps de tout être humain dont ils ont causé la mort. Le lien physique le plus dangereux entre mère et enfant est le mamelon de la mère. Après les funérailles du petit Waniol, un jeune homme nommé Chris me conta que traditionnellement, quand une mère qui allaite son enfant meurt, ses mamelons doivent être coupés et déposé au plus haut d'un arbre. De cette façon, l'âme du bébé, qui reste attachée aux mamelons, ne sera pas elle aussi attaquée par les mauvais esprits et les sorcières qui sont supposés avoir mangé le corps de la mère.

Deux jours après les funérailles du petit Waniol, deux hommes, Yalowad et Nonaino, accompagnés de deux des fils de Yalowad, Yon et Saiwan, passèrent chez moi et me dirent que des empreintes de pas humains avaient été repérées dans les cendres, près de la tombe de Waniol, ce qui tendait à prouver que les sorcières étaient venues la nuit dans l'espoir de manger le corps. Pour cette raison, Wani, le père du garçon était en train de construire une maison miniature au-dessus de la tombe pour protéger le cadavre. Je questionnai le groupe à propos de la pratique qui consiste à couper les mamelons de la mère décédée. Yalowad me dit que cela se pratiquait autant avant le contact, quand on exposait les cadavres à l'extérieur, qu'à l'époque actuelle, où l'on pratique l'inhumation chrétienne. Il remarqua que ce ne fut pas fait dans le cas de la mère de Waniol, mais que le garçon avait grandi sans problèmes de toute façon. Nonaino ajouta que les mamelons devaient être déposés sous l'eau, dans le creux d'un arbre, de façon à ce que les fourmis et autres insectes ne puissent les manger. Si les fourmis étaient capables de les manger, le bébé tomberait malade ou mourrait.

Quatre jours après la mort de son fils, Pastor Wani vint chez moi. Suivirent Wason, sa plus jeune fille, et Madfe, le vieil homme qui m'avait vendu l'étui pénien de son père. Je m'apprêtais à essayer de faire du gâteau de sagou pour la première fois, alors Wani décida de m'aider. Nous émiettâmes le sagou dans un pot, ajoutâmes un peu d'eau froide, et délayâmes le mélange. Nous versâmes ensuite beaucoup d'eau bouillante, du lait de coco et du sel, et remuâmes l'ensemble jusqu'à ce qu'il épaississe. C'était délicieux.

Wani déclara qu'il s'inquiétait de vieillir sans avoir un fils pour s'occuper de lui. Je l'interrogeai à propos de sa fille, et il répondit que les filles étaient différentes - elles se marient et s'en vont, alors qu'un vrai fils reste avec son père et le nourrit. Il conta qu'il avait rencontré, dans le voisinage d'Eliptamin, un vieil homme sans enfants qui était très malheureux car les gens ne lui donnaient que très peu de nourriture. Il lança, en plaisantant, qu'il quitterait le village pour une longue période afin d'oublier. Dix jours après la 
mort de son fils, Wani se retira une nuit dans une case de jardin isolée pour rompre avec son habitude de rester dans la maison à ne rien faire. Il expliqua qu'il ne voulait plus parcourir les chemins qui lui étaient familiers car il voyait partout les entailles dans les arbres que Waniol faisait quand il se promenait avec sa machette à la main. Ces marques, comme toute l'organisation du village, ravivaient les douloureux souvenirs de son fils. Elles étaient des mémentos malvenus, contrairement aux souvenirs bienvenus que les gens veulent conserver d'une personne une fois que la douleur causée par la perte est passée. Plus d'un an après, quand il fut enfin prêt, Wani me demanda dans une lettre de lui envoyer une photo de son fils.

Un mois et demi après le décès, j'étais en train de parler avec Yon, un garçon du village, quand il désigna soudain les vêtements de Waniol accrochés en haut d'un arbre. C'est Pastor Wani, le père de Waniol, qui les avait accrochés là. Yon suggéra qu'il l'avait probablement fait pour empêcher les mauvais esprits de s'attarder autour de la maison. Huit mois plus tard, les vêtements étaient toujours là et je trouvais une occasion pour demander en personne à Pastor Wani pourquoi il avait fait cela. Il répondit :

«Quand les vêtements seront complètement décomposés, je pourrai oublier. Cela n'a rien de traditionnel, c'est juste une idée à moi. »

Dans ce petit récit, on voit que Wani tient tête à son désir conflictuel d'à la fois s'accrocher à son fils et de l'oublier. Au début, il évitait les lieux qui lui rappelaient son fils, puis il suspendit un puissant mémento, hors du champ de vision habituel, mais malgré tout visible d'un seul coup d'œil vers le haut. Là-haut, ce mémento pouvait être la plupart du temps ignoré, mais on pouvait aussi le contempler à l'occasion, et sa lente décomposition marquait l'accalmie graduelle du douloureux souvenir de la perte. Il est aussi possible que les explications de Yon comportent une part de vérité. Les mamelons d'une mère décédée sont déposés dans l'eau sous les racines d'un arbre où ils peuvent se décomposer en étant à l'abri des esprits. Similairement, il se peut que les vêtements de Wani aient été accrochés dans l'arbre jusqu'à ce qu'ils se décomposent tout en étant maintenus à l'écart, hors de portée des esprits. Mais il semble qu'il s'agissait de l'extension par un enfant de croyances traditionnelles sur le pouvoir spirituel des reliques corporelles et des mémentos, interprétation qui exprime de façon simplifiée la gestion des souvenirs.

\section{La destruction immédiate : le pandanus d'une jeune mère}

Un matin de juin 1995, j'entendis gémir dans le village de Yakob. Sekina, femme de Muluasi et mère de six enfants, était morte à dix heures la veille au soir. Elle avait fait une chute quelques jours auparavant en portant un lourd sac dans la forêt et son état s'était progressivement détérioré. Son frère, Salowa, me conta qu'après sa chute, une nuit, alors qu'elle était sortie de la maison pour se soulager, elle entendit des rats couiner puis sentit subitement le vent, et un frisson de peur l'envahit. Elle appela alors Jésus à l'aide en criant. Quand elle rentra dans la maison, son état empira. Elle se plaignait d'une douleur à la poitrine, forte comme si une lame y était logée. Elle se mit alors à penser que des esprits malins avaient tiré une flèche sur elle. Salowa ajouta que personne ne savait vraiment ce qui avait causé la mort de sa sœur, mais que c'était le seul événement dont il pouvait se souvenir pour l'expliquer. Au cours de la journée, on construisit son cercueil en planches alors que son corps reposait dans la maison communautaire.

Les funérailles eurent lieu le jour suivant. Le matin, de jeunes hommes creusèrent la tombe. Le cercueil consistait en une boîte avec des pieds, faite de planches découpées à la scie et couverte d'étoffes colorées. Il était placé près du centre de la maison. L'après-midi, on servit de la nourriture et les gens entraient et sortaient de la maison. Peter, le frère de Sekina, passa quelques instants à la tête du cercueil, suivi par sa camarade Onai, qui se mit à sangloter puis à chanter le deuil. Muluasi, le veuf de Sekina, était assis dans le coin, avec ses enfants. Il posait de temps en temps sa tête entre ses mains ou s'allongeait sur le côté, l'air fatigué.

Les Asabano ont tendance à être très fatalistes vis-à-vis de la mort et, lors des funérailles, ils pleurent moins que leurs voisins saiyo (des Sisimin). Tout le monde semblait éprouver beaucoup plus de chagrin pour cette mort en particulier. Néanmoins, tout n'était pas que tristesse et il $\mathrm{y}$ avait parfois aussi des sourires et des plaisanteries. Alors que j'étais assis avec les autres hommes dans la maison, Peter commença à tresser mes longs cheveux. Les femmes étant assises en groupe en face de nous.

Finalement, quelqu'un sonna la cloche de l'église de cette fervente communauté baptiste et d'autres personnes entrèrent. Roti, le pasteur téléfolmin, fit chanter un cantique vernaculaire, puis se mit à lire des passages de la Bible de façon très pathétique. Il parla de la temporalité de la vie et de la nécessité de croire pour que, une fois 
morts, nous puissions aller au paradis. Le pasteur se référa à Sekina, laissée aux mains de l'ennemi par Dieu qui avait décidé que son heure était venue. Il demanda ensuite si quelqu'un voulait dire quelque chose et Peter fit un discours valorisant encore plus l'importance du christianisme. Le service se termina après une prière de groupe. De jeunes hommes attachèrent avec des lianes une perche tendue en long au-dessus du cercueil et le soulevèrent. Pendant ce temps, des personnes pleuraient et, pensant à cette généreuse et gentille jeune femme qui allait tant manquer à sa famille, je me mis finalement à pleurer moi aussi. Quelqu'un s'exclama en Asaba que l'homme blanc pleurait aussi et, à ce moment, la maison entière éclata en pleurs. Nous restâmes tous un bon moment dans cet état pitoyable.

Du liquide avait apparemment coulé du cercueil, ce pourquoi quelqu'un versa des cendres du foyer sur le sol là où cela s'était produit. Un peu plus tard, une femme âgée du nom de Yaiyo, connue comme une Femme-esprit - un medium du Saint-Esprit - versa de l'eau bouillante sur la portion de sol où était posé le cercueil. Lentement, les gens commencèrent à s'en aller. Je fis finalement de même et suivit Peter qui était en pleurs sur les marches menant à la véranda.

Deux jours après l'enterrement, un repas festif fut organisé en l'honneur de Sekina. Les hommes tuèrent trois cochons, dont un lui ayant appartenu, et les mirent à cuire dans un four semi-enterré. Pendant l'après-midi, un jeune homme appelé Mosibo coupa un très beau pandanus rouge (Tok Pisin : marita; Pandanus conoideus) sous les yeux de Muluasi, le veuf de Sekina. Muluasi expliqua que sa femme avait planté cet arbre pour sa famille, mais que maintenant qu'elle était partie, il valait mieux le détruire. Le pandanus rouge offre une nourriture prisée. On fait une sauce de ses fruits rouge vif et riches en huile. Dans le temps d'avant le contact, il figurait dans les mythes et les rituels ésotériques comme un symbole de la femme, lié aux menstruations (Lohmann, 2000a : 100), ce qui doit lui avoir donné une signification particulièrement forte comme symbole de la maternité nourricière qui avait été perdue avec la mort. Toutefois, personne ne me suggéra cette association. Plus tard dans la journée, je demandais à Tona, la femme de Mosibo, pourquoi un si bel arbre avait été détruit. "C'est notre coutume », répondit-elle. « Notre sœur est terminée, donc ses choses sont terminées aussi. » Mosibo était lointainement lié à la défunte par mariage. J'étais assez perturbé par ce qui me semblait être un acte de destruction capricieux, mais en savoir les véritables raisons me rendit d'autant plus triste. Il me semblait que cet arbre aurait pu être une importante source de nourriture pour le futur, un héritage laissé par cette dame chérie qui était morte. Cela eut été une façon pour elle de continuer à pourvoir aux besoins de sa famille et je m'imaginais qu'on aurait pu le laisser grandir en souvenir de sa bonté. Mais, conformément à la coutume, et pour des raisons que les gens ne pouvaient formuler explicitement, l'arbre n'était pas supposé être conservé comme mémento.

Les arbres fruitiers ne sont habituellement pas détruits quand leurs propriétaires meurent - on se réfère souvent avec fierté et affection aux bosquets de pandanus et de sagou comme ayant été plantés par un ancêtre. Néanmoins, l'acte de détruire un pandanus lors d'un deuil est documenté dans la région d'Anga en Papouasie Nouvelle-Guinée (Bonnemère, 1996 : 154-155; Lemonnier, 2006 : 152). Lemonnier (ce volume) signale que les nouveaux arrivants sur les lieux d'une veillée mortuaire détruisent des pandanus rouges et d'autres plantations dans les jardins ankave. L'abattage du pandanus de Sekina, tout comme le fait que Wani suspendait les vêtements de son défunt fils, ne correspondait pas simplement à des pratiques coutumières. Il s'agissait d'un acte à la fois unique et congruent avec la culture asabano. Il s'agissait d'actions extrêmes entreprises comme stratégies pour exprimer une émotion et pour gérer le souvenir en prenant en charge le cours de la mémoire. Dans le cas de Sekina, le chagrin de ceux qui lui survivaient était d'autant plus grand que sa mort était prématurée et qu'il s'agissait d'une personne fidèlement généreuse. Peut-être était-il trop horrible de se voir périodiquement rappeler cette perte chaque fois que l'arbre porterait des fruits? Une séparation plus complète était nécessaire.

Presque deux semaines après l'enterrement, le veuf Musuasi me dit que lui, ses enfants et son cousin Madfe (qui m'avait vendu l'étui pénien de son père) envisageaient d'aller s'installer au village de Paupe, près de la rivière Frieda, dans une autre région linguistique où ils avaient des parents. Il ne voulait pas rester au hameau de Yakob à Duramin après la perte de sa femme, de peur que ceux qui avaient causé la mort de sa femme ne prennent de nouvelles vies. De plus, il ne voulait pas se souvenir d'elle dans un environnement qui était devenu un mémento non souhaité. Ce même jour, de nombreuses personnes vinrent pour construire un enclos à cochon autour d'un nouveau jardin que Sekina avait planté. Muluasi expliqua que, contrairement au pandanus, "[il] ne [voulait] pas jeter ça. Les autres et [lui devraient] pouvoir s'en nourrir ». 
Alors que l'arbre fruitier de Sekina fut détruit et son cochon mangé, son jardin fut donc préservé et soigné. L'arbre qui était supposé constituer le souvenir le plus durable fut facilement détruit à la hache. Le cochon aurait pu servir de mémento vivant, mais il fut tué pour nourrir la famille et les amis de Sekina rassemblés pour lui rendre hommage. Le jardin récemment planté, qui aurait pu jouer le rôle de souvenir jusqu'à la récolte, était plus difficile à détruire et fut finalement entretenu et sarclé. Dans ce cas où une mort frappa particulièrement fort l'ensemble de la communauté, il y eut une tendance plus forte qu'à l'habitude à détruire un mémento durable. Les gens souhaitaient que la triste nostalgie de la vie passée avec Sekina s'efface rapidement et ils souhaitaient exprimer la rage et la désolation provoquées par sa perte. Ainsi, le déplacement de son mari et de ses enfants et la destruction de sa propriété survinrent quand l'ensemble de l'environnement du village était devenu un mémento importun de la disparition de Sekina.

\section{Machettes, ficelles et empreintes de mains : stra- tégie économique et valeur sentimentale}

En 2005, dix ans après la mort de Sekina, je retournai avec ma femme, l'archéologue Heather M.-L. Miller, en visite à Duranmin pour un mois. Au cours de ce bref séjour, je vis plusieurs exemples indiquant que l'usage des mémentos demeurait bien établi.

Un matin, Belok, un vieil ami et informateurclef, nous rendit visite à la maison. J'avais apporté des présents, de ceux dont je me souvenais que les gens appréciaient, et Belok demanda une machette. J'étais heureux de lui en offrir une du plus beau des modèles que j'avais apportés. Il la reçut avec un large sourire et un plaisir manifeste. Il souleva une rame de palme du plancher et la dissimula au-dessous. Il déclara que notre hôte, Laurie, qui nous laissait l'usage de sa maison vacante, la lui donnerait après notre départ. Il évitait ainsi la jalousie de la part des autres, ceux qui n'avaient pas reçu un présent si délicat. Cependant, il sembla trop impatient pour attendre et revint le soir même, au crépuscule, pour la récupérer.

Belok identifia immédiatement la machette comme un memori ou mémento de moi. À n'en pas douter il voulait m'épater en signifiant qu'il allait en prendre soin et qu'il penserait à moi quand il l'utiliserait. Ceci montre que le statut de mémento peut être invoqué pour aider à justifier des réclamations envers des objets de valeur dont on hésite à se séparer. D'un autre côté, identifier la machette comme un mémento lui permettait de se défendre contre ceux qui allaient perpétuellement la lui réclamer en cadeau, ou comme mémento de lui-même. La réciprocité est quelque chose de très fort et d'omniprésent dans la société asabano et l'on doit sans cesse en assumer les coûts autant que les bénéfices. Étiqueter un objet comme le mémento de quelqu'un est un argument fort - si ce n'est une garantie absolue contre la demande de partage de tout bien. Dans ce sens, conférer le statut de mémento à quelque chose a une fonction qui est assurément autant économique que sentimentale : un memori peut difficilement être réclamé par les autres. L'observation de ce cas me rappela un autre exemple dans lequel j'avais été impliqué en 1995. Un ami $\mathrm{du}$ hameau de Yakob nommé Sokiale m'emprunta un petit sac à dos pour un séjour de chasse et de jardinage dans la forêt. Il ne revint finalement pas au hameau et emporta mon sac à dos avec lui dans une autre région. Quand j'eus de nouveau besoin du sac, je lui fis parvenir un mot lui demandant de me le renvoyer. Ceux qui lui passèrent le message me contèrent qu'il refusait de s'en séparer, affirmant qu'il s'agissait d'un memori de moi. Pour ne pas me laisser amadouer, ni me faire escroquer d'un élément nécessaire à mon équipement de terrain, je renvoyai une réponse contestant le statut de mémento du sac à dos, précisant que je ne l'avais pas donné de façon permanente (j'avais été très clair avec lui sur le point qu'il s'agissait d'un prêt) et que, de surcroît, j'en avais besoin pour mon travail. Le sac me fut renvoyé sur le champ. Cet incident illustre le fait que, puisque les mémentos ont une valeur sentimentale, les propriétaires d'objets particulièrement précieux peuvent justifier de cette valeur pour se défendre des réclamations. De telles revendications d'inaliénabilité sont sujettes à négociations et peuvent échouer, mais elles offrent un argument de défense morale face à une demande de réciprocité tout aussi morale.

La distinction entre valeur utilitaire et symbolique est importante d'un point de vue heuristique, bien que l'une vienne rarement sans l'autre (une introduction à ce sujet fascinant est proposée par Michael Fotiadis, 1999 : 386-387). Tous les objets, même les objets symboliques sans usage utilitaire évident, ont une fonction précise. Les exemples fournis jusqu'alors concernaient tous des objets qui possèdent une valeur économique fondée sur une désirabilité utilitaire et transférable, indépendante de la valeur symbolique, unique, subjective et sentimentale qui a été superposée au-dessus de ces autres dimensions par leur désignation comme mémento. Les deux objets dont parlent les exemples suivants four- 
nissent un contrepoint intéressant car ils n'ont virtuellement aucune valeur économique dérivant de leur usage non sentimental. Leurs propriétaires ne les désignaient pas comme mémentos pour éviter les éventuelles réclamations, mais simplement pour signifier leur valeur sentimentale. Un jour, à la fin de juillet 2005, Sablaio, un homme âgé mais vigoureux du village de Yakob, que je connaissais bien depuis mes précédents séjours sur le terrain, nous montrait, à Heather et à moi, les outils avec lesquels il fabriquait des flèches. En déballant un baluchon, il expliquait que le vieux morceau de ficelle défraîchie qui maintenait ensemble les morceaux d'étoffes du baluchon avait été fait par feue sa femme Kola, qui avait été exécutée pour sorcellerie quelques décennies plus tôt: "C'est un mémento de la mère de Sansib », expliqua-t-il, mentionnant sa fille connue comme supporter énergique de l'Église baptiste.

Un peu plus tard, Sansib montra à Heather un morceau d'ocre rouge qu'elle avait utilisé pour colorer le même type de cordelette, qui est encore aujourd'hui fabriquée par les femmes à partir d'écorce de tulip (Gnetum gnemon) pour les sacsfilets. Elle nous conta que cette ocre avait aussi appartenu à sa mère. Elle ne semblait pas éprouver autant d'attachement sentimental à ce morceau d'ocre que son père en éprouvait pour son morceau de ficelle. Elle offrit de le briser en deux pour le partager avec Heather. Heather refusa, peut-être plus troublée par la valeur sentimentale de l'objet que ne l'était Sansib elle-même.

Avec mon esprit obnubilé par la façon dont les objets évoquent les souvenirs, je remarquais avec une nouvelle attention des empreintes de mains faites avec des cendres mouillées sur le mur du fond de la traditionnelle maison communautaire et des maisons familiales individuelles devenues communes depuis le contact. Un jour, un garçon nous apporta une note écrite par un jeune père nommé Mason, un bon camarade et informateur lors de mes terrains précédents, nous informant qu'il était malade et demandant que je lui apporte des médicaments. Heather et moi marchâmes jusqu'à sa maison où nous le trouvâmes en nettement meilleur état que nous l'imaginions, souriant et de bonne humeur, apparemment en voie de guérison d'une crise de malaria. Nous lui offrîmes un cachet d'aspirine contre son reste de mal de tête et discutâmes amicalement avec lui et sa femme Maria, pendant que ses enfants jouaient autour de nous. Je vis des empreintes de mains sur le mur de sa maison et le questionnai à ce propos. Mason me dit qu'elles étaient des ol memori, des mémentos, des gens qui avaient visité sa maison. Il en désigna une faite par Yalomo, un homme que j'avais connu au cours de mon dernier terrain et qui était mort récemment. « Maintenant, quand je les regarde, je pense fort à lui ». Les empreintes de main n'ont pas de valeur économique et, que je sache, ne sont jamais réclamées ni échangées. Pourtant, elles ont le pouvoir d'évoquer la mémoire d'un autre; cette empreinte de la main de Yalomo était supportable et même apparemment poignante et enrichissante dans la maison de Mason. En 2005, on continuait de fabriquer des souvenirs et de les gérer au travers de mémentos, entrelaçant le chagrin et l'affection, l'économie et les sentiments, l'idéel et le matériel.

\section{Conclusion : les mémentos comme reflets des changements de la mémoire}

La mémoire est intensifiée par la perception et contrôlée par la manipulation d'objets matériels. Dans les cas décrits ici, les mémentos des défunts servaient de témoignage de la réalité passée de la présence physique vivante d'une personne et de ses interactions sociales avec le survivant. Ils renforçaient et offraient une manifestation substantielle du souvenir éthéré. Les humains conservent des objets pour se rappeler des gens, des lieux, des expériences dont ils sont séparés, soit par le temps, soit par l'espace. Quand certains souvenirs ne sont pas considérés comme importants ou ne sont pas menacés d'être oubliés, les gens se débarrassent sans considération d'objets qui étaient ou auraient pu devenir des mémentos. Lorsque les souvenirs sont un fardeau et que les gens cherchent à oublier ou veulent soulager leur chagrin, les mémentos peuvent être détruits, graduellement ou rapidement, selon l'empressement que l'on éprouve à s'en débarrasser. Prendre soin d'un mémento entretient le souvenir. Détruire un mémento aide à oublier. Le vendre sert à remercier ou à accepter d'oublier.

Quand un décès est encore un souvenir frais et vif, les endeuillés doivent endosser les nouvelles associations et les nouvelles significations associées aux mémentos qu'ils possédaient déjà ou dont ils vont hériter. En ces moments de sentiments agités et changeants, et de significations qui sont associées ou détachées aux mémentos, l'ambivalence peut conduire à des actions sévères, comme la destruction d'un arbre fruitier. L'instabilité de la condition émotionnelle peut aussi engendrer des rêves difficiles, comme je le rappelle dans un autre cas étudié en 1995. Cette situation fournit aussi un éclairage sur la croyance en cours chez les Asabano, selon 
laquelle, lorsque les défunts sont physiquement morts et incapables d'entrer en contact dans une relation entière avec des compagnons, ils peuvent néanmoins continuer de «vivre» spirituellement et d'interagir avec les gens (pour d'autres exemples, voir Lohmann, 2003a). Martin, un jeune homme d'environ 18 ans à l'époque où je l'avais interviewé, me conta ses mésaventures avec une flèche-mémento qu'il avait héritée de son " oncle» Agatai, qui avait surpris tout le monde dans la communauté en se pendant, apparemment sans raison.

«Quand j’étais à Téléfomin [inscrit au lycée], je fis le rêve que Agatai était mort. Il était mon oncle. Je [lui] dis : "Cette flèche qui t'appartenait est désormais à moi". Il répondit : "Non, elle m'appartient". Je répondis : "Tu es mort maintenant, pourquoi veux-tu garder ta flèche?". Il s'énerva et me poussa. Je crus que c'était réel et me réveillai. Je pensais qu'il était vraiment venu me parler. Ce n'était pas seulement mon imagination $»$.

Le mémento, dans ce cas, avait acquis un fâcheux aspect : son défunt propriétaire voulait le récupérer. Les circonstances étranges entourant sa mort ont pu provoquer de l'insécurité dans les souvenirs que Martin gardait de leur relation et une certaine préoccupation relative au fait de savoir s'il était juste ou non de prendre cette flèche. Cependant, connaître les détails de l'inspiration émotionnelle sousjacente à ce rêve nous est impossible sans une connaissance plus profonde de Martin à ce moment, comme Melford Spiro (2003) le souligne au sujet des limites de l'étude ethnographique des rêves. Cette saynète illustre néanmoins que les mémentos des défunts peuvent prendre des sens nouveaux et provoquer de nouvelles associations de la mémoire, même après que la personne à laquelle ils renvoient est biologiquement morte.

De façon similaire, Stephen Leavitt (1995) écrit au sujet d'un cas arapesh dans lequel un fils endeuillé interpréta la non-apparition du cargo comme un signe d'abandon de la part de son défunt père, soulignant non seulement le lien entre les objets et le deuil, mais également la possibilité que les objets apparaissant après la mort d'une personne puissent prendre la fonction de mémentos. Quand vient la mort, des conflits s'installent dans l'esprit des endeuillés. Il y a simultanément un désir de se rappeler des défunts en gardant des objets servant de souvenirs et un désir de supprimer ces mêmes objets, de façon à ce que les souvenirs et les sentiments persistants d'attachement teintés de perte ne fassent pas de dommage à une saine socialité chez les survivants. Les Asabano affrontent ce paradoxe moral en alternant la destruction ou les soins portés des biens des morts, selon les circonstances et avec une bonne marge de flexibilité et de liberté suivant les émotions personnelles du moment. Ces façons de manipuler les souvenirs des morts ont des effets distincts sur le rappel à la mémoire du moment et sur les expériences sensorielles évocatrices du défunt. La signification des mémentos dépend de la subjectivité d'une personne particulière dans un contexte culturel particulier. Sorti de ce contexte, la signification et la fonction commémoratives de ces objets sont perdues. Ainsi, chacun des cas considérés ici dépeint une stratégie unique dans la pratique d'acteurs individuels, mais cette stratégie est néanmoins également intelligible à l'intérieur d'un milieu culturel asabano partagé (Bourdieu, 1977).

Les souvenirs changent. Les images mentales des gens et des événements du passé sont colorées et altérées par des expériences et dans des contextes changeants. Ceux-ci incluent l'entourage social et culturel, les événements historiques et l'environnement physique. Enchâssés dans celui-ci se trouvent des objets fabriqués, des « objets » non modifiés (des arbres, par exemple), des paysages et des lieux signifiants qui, tous, existent physiquement dans le monde externe et sont symbolisés dans les esprits en recevant toutes sortes de représentations et valeurs (Lohmann et Sered, 2007; Sperber, 1996). «In many cases», Daniel Miller (1998:5) a observé que «material culture is better identified as a means rather than an end». Le présent article portait sur l'un de ces moyens: les mémentos sont une catégorie d'éléments de la culture matérielle qui sont utilisés par leurs détenteurs pour gérer leurs souvenirs ( $c f$. Walker, 2001). Les individus utilisant les mémentos de cette façon sont confrontés à des choix : quel objet utiliser comme mémento? Les mémentos devraient-il être conservés ou détruits et comment devraient-ils l'être ? Ces choix sont semblables aux décisions technologiques qui ont façonné les choix de la culture matérielle parmi les différentes cultures et tout au long de l'histoire humaine et de la préhistoire (voir Lemonnier, 1993 ; Miller, 2007). J'ai exploré ici un cas ethnographique d'objets qui évoquent des souvenirs. Certains mémentos font resurgir des souvenirs de façon involontaire dans ce que l'on pourrait appeler un rappel autonome ( $c f$. Stephen, 1989). D'autres sont utilisés pour cajoler et nourrir des souvenirs renforcés intentionnellement par la réinterprétation et l'imagination. $\mathrm{Ce}$ qui arrive au mémento est le reflet de ce qui est 
arrivé ou de ce que l'on aimerait qu'il arrive à la mémoire qu'il représente.

Comme l'observait Edward B. Tylor il y a longtemps, «imagination keeps together the personality and the relics of the dead» (1877 [1871] : 151). En considérant les mémentos des défunts, nous sommes en présence de l'usage que les gens font de leur imagination pour projeter une substance apparente et une signification sur des objets dans le monde empirique. Cette projection imaginative dessine alors les expériences sensibles ultérieures de ces objets, rappelant à l'esprit les gens et les événements disparus. Les gens utilisent les mémentos pour les aider à se souvenir, mais ces mêmes mémentos peuvent aussi rappeler à la mémoire des souvenirs malvenus. Quand il s'agit de mémentos de défunts, souvenir et oubli sont impliqués dans des proportions souvent changeantes. Mon argumentation dans cet article portait sur le fait que les Asabano, comme toute autre personne, utilisent et manipulent activement des mémentos pour gérer des souvenirs de façon intentionnelle, encourageant ce qui est plaisant et détruisant ce qui est déplaisant. Dans ce sens, la manipulation des mémentos peut être interprétée comme une technique de gestion de la mémoire.

\section{BIBLIOGRAPHIE}

BonNEMÈre Pascale, 1996. Le pandanus rouge: Corps, différence des sexes et parenté chez les Ankave-Anga (Papouasie Nouvelle-Guinée), Paris, CNRS/Éditions de la maison des sciences de l'homme.

Bourdieu Pierre, 1977. Outline of a Theory of Practice, Cambridge, Cambridge University Press.

Csikszentminalyi Mihaly, 1993. Why We Need Things, in S. Lubar and W. D. Kingery (eds), History from Things: Essays on Material Culture, Washington, Smithsonian Institution Press, pp. 2029.

Dalton Doug, 2001. Memory, Power, and Loss in Rawa Discourse, in J. M. Mageo (ed), Cultural Memory: Reconfiguring History and Identity in the Postcolonial Pacific, Honolulu, University of Hawai'i Press, pp. 105-129.

Fotiadis Michael, 1999. Comparability, Equivalency, and Contestation, in J. E. Robb, (ed), Material Symbols: Culture and Economy in Prehistory, Carbondale, Center for Archaeological Investigations, Southern Illinois University, Occasional Paper 26, pp. 385-398.

GODELIER Maurice, 1999 [1996]. The Enigma of the Gift, N. Scott (trans), Chicago, University of Chicago Press.
Graeber David, 2001. Toward an Anthropological Theory of Value: The False Coin of Our Own Dreams, New York, Palgrave.

Hallam Elizabeth et Jenny Hockey, 2001. Death, Memory, and Material Culture, Oxford, Berg.

Hertz Robert, 1960 [1907]. A Contribution to the Study of the Collective Representation of Death, Death and the Right Hand, Glencoe, Il, Free Press, pp. 29-88.

LeavitT Stephen C., 1995. Seeking Gifts from the Dead: Long-Term Mourning in a Bumbita Arapesh Cargo Narrative, Ethos 23, pp. 453-473.

Lemonnier Pierre (ed), 1993. Technological Choices: Transformations in Material Cultures since the Neolithic, London, Routledge.

Lemonnier Pierre, 2006. Le sabbat des lucioles : Sorcellerie, chamanisme et imaginaire cannibale en Nouvelle-Guinée, Paris, Stock.

Lohmann Roger Ivar, 2000a. Cultural Reception in the Contact and Conversion History of the Asabano of Papua New Guinea, Department of Anthropology, University of Wisconsin-Madison, Ph.D dissertation.

—, 2000b. The Role of Dreams in Religious Enculturation among the Asabano of Papua New Guinea, Ethos 28, pp. 75-102.

—, 2001. Introduced Writing and Christianity: Differential Access to Religious Knowledge among the Asabano, Ethnology 40, pp. 93-111.

—, 2003a. Supernatural Encounters of the Asabano in Two Traditions and Three States of Consciousness, in R.I. Lohmann (ed), Dream Travelers: Sleep Experiences and Culture in the Western Pacific, New York, Palgrave Macmillan, pp. 188-210.

—, 2003b. Turning the Belly: Insights on Religions Conversion from New Guinea Gut Feelings, in A. Buckser et S. D. Glazier (eds), The Anthropology of Religious Conversion, Boulder, Rowman and Littlefield, pp. 109-121.

-, 2005. The Afterlife of Asabano Corpses: Relationships with the Deceased in Papua New Guinea, Ethnology 44, pp. 189-206.

—, 2007. Sound of a Woman: Drums, Gender, and Myth among the Asabano of Papua New Guinea, Material Religion: The Journal of Objects, Art and Belief 3, pp. 88-109.

Lohmann Roger Ivar et Susan Starr Sered, 2007. Introduction: Objects, Gender and Religion, Material Religion: The Journal of Objects, Art and Belief 3, pp. 4-13.

Miller Daniel, 1998. Why Some Things Matter, in D. Miller (ed), Material Cultures: Why Some Things Matter, Chicago, University of Chicago Press, pp. 3-21.

MiLler Heather Margaret-Louise, 2007. Archaeological Approaches to Technology, Amsterdam, Academic Press. 
SPERBER Dan, 1996. Explaining Culture: A Naturalistic Approach, Oxford, Blackwell.

SPIRo Melford E., 2003. The Anthropological Import of Blocked Access to Dream Associations, in J. M. Mageo (ed), Dreaming and the Self: New Perspectives on Subjectivity, Identity and Emotion, Albany, State University of New York Press, pp. 165-173.

StePHEN Michele, 1989. Self, the Sacred Other and Autonomous Imagination, in M. Stephen and G. Herdt (eds), The Religious Imagination in New Guinea, New Brunswick, Rutgers University Press, pp. 41-64.
Tylor Edward B., 1877 [1871], Primitive Culture: Researches into the Development of Mythology, Philosophy, Religion, Language, Art and Custom, New York, Henry Holt and Company.

WALKer William H., 2001, Ritual Technology in an Extranatural World, in M. B. Schiffer (ed), Anthropological Perspectives on Technology, Albuquerque, University of New Mexico Press, pp. 87-105. 
